\title{
Method of the Finned Pipe Heat Exchanger Modelling using Porous Medium
}

\author{
Nikolai Efimov-Soini, Mikhail Kiauka, Aleksey Borovkov \\ Peter the Great St. Petersburg Polytechnic University \\ St. Petersburg, Polytechnicheskaya, 29, Russia \\ Efimovs_nk@spbstu.ru; kiauka_myu@spbstu.ru; borovkov@spbstu.ru;
}

\begin{abstract}
This paper proposes the method of finned pipe heat exchanger modelling using porous medium approach. In this approach fins are changed to the porous medium by means of a formal algorithm using ANSYS CFX. The last one permits to develop heat exchanger design take into account the temperature and pressure fields. Then the modelling results can be used for the stress analysis and in the construction optimization process. The paper scope is to introduce a simple, cost-efficient method, which is capable to simulate a gas flow of a heat exchanger and can be integrated into more complex simulations.
\end{abstract}

Keywords: Porous medium, Heat exchanger, CFD, Heat transfer, ANSYS CFX

\section{Introduction}

A heat exchanger is a widely used device that exchanges heat between two fluids or gas. Many types of heat exchangers used in steam power plants, chemical manufacturing, atomic plants etc. The design of heat exchangers is a complicated problem. It takes into account not only heat-transfer analysis alone, but the cost of fabrication and installation, weight, size etc. In many cases, although cost is an important consideration, size and footprint often tend to be the dominant factors in choosing a design.

Usually developers design the heat exchanger using empirical correlations. On the other hand, one of the most dynamically improving research field is the Computational Fluid Dynamics (CFD), which is a good alternative of the expensive and time demanding experimental test measurements in wind tunnels or laboratories. Meanwhile the simulation methods often are not just a more cost efficient solution than experimental measurements, but even provide more details and information about a given problem.

Analytical approaches permit to calculate average values, but not the local values. Meanwhile, the CFD calculation permits to calculate the local values, but this method can be very expansive for complex products. Likewise, there are commercial software to calculate not only CFD, but also the stress analysis and optimization calculation. It means is possible to use one model for all types of simulation with need to design product. Although numerous semi-empirical correlations are available in the literature for estimating the convection coefficient of tube bundles with solid fins (both annular and helically wound), the values predicted by such correlations rarely agree with one another, and in some cases can differ by as much as $30 \%$ [1].

Currently, the use of empirical correlations is an essential element of heat-exchanger design and modelling. Walter and Hofmann modelled boiler dynamics [2], demonstrating the effect of different correlations for external Nusselt numbers on predicted boiler behaviour. Martinez et al. [3] investigated the optimum fin tube geometry, based on published correlations for Nusselt number and pressure drop. Prieto et al. [4], [5] employed empirical correlations to predict temperature distribution in unfinned boiler tubes.

Suggested paper propose the engineering method of heat exchanger modeling by means porous medium. Similar method use Hoomen and Gurgenci for air cooling condensers simulation [6]. The finned tube bundles in the condenser are represented by a porous matrix, which is defined by its porosity, permeability, and the form drag coefficient. The porosity is equal to the tube bundle volumetric void fraction and the permeability is calculated by using the Karman-Cozney correlation. On the other hand the similar approach is used in wavy fin-and tube intercooler [7]. Therefore, the real intercooler geometry is used except for the wavy fins, which are substituted with the porous media model in order to 
decrease the computing quality. Analyzing flow could make calculations to predict flow resistance characteristics using the CFD method. In this study, the heat transfer between fluid domain and solid domain was calculated conjointly. Analyzing flow characteristics could make calculations to predict flow resistance characteristics using the CFD method for the porous model. In this case the difference between experimental results and calculation is $10-15 \%$. Also the porous medium simplification use Imke [8] for of single- and two-phase flow heat transfer microchannel heat exchanger. In this case the solid structure is represented as a porous medium with its own temperature field. Wall friction, heat transfer between structure and fluid, and the momentum coupling between the fluid phases are modelled by conventional empirical correlations. To calculate the automobile radiator the porous medium approach is used and hydrodynamic and thermal characteristics are obtained [9] [. Numerical simulations have been carried out to investigate the thermodynamic characteristics of a full-size plate-fin heat exchanger via the porous medium approach [10], [11],. Porous medium approach to model interlayer-cooled 3d-chip stacks is used and validated [12].

In this paper we propose the engineering method of heat exchanger calculation, when tube fins can be represented as a porous media, and tube wall is solid domain. Thus, this method permits to transfer of pressure and temperature fields to mechanical models for stress analysis of tubes. Our results are both theoretical and applied in industrial cases.

The main difference of the method to other porous equivalent methods is in transformation to porous medium only finned region. Thus, the geometry of the tube is not changed. It means the finite element model can be used for different types of analysis - for example heat transfer, CFD, stress analysis and other. Also, the user can use one software (e.g. ANSYS Workbench) for all types of analysis.

\section{Porous medium fundamentals}

For heat exchanger calculation we used ANSYS CFX. In ANSYS CFX the flow in porous media can be simulated by using either of two models [13]:

- A fluid domain together with a momentum loss model. In this case the porosity is accounted for only through this loss term, other terms in the governing equations are not changed. In ANSYS CFX this formulation is called the "superficial velocity formulation".

- A domain that involves one or more fluids and an optional solid. All the terms in the governing equations are modified by the porosity, as well as the loss term. In ANSYS CFX this formulation is called the "true velocity formulation", or the "full porous model".

At the moment in our research we use the full porous model is based on the commonly used Darcy's law for pressure drop determination, and on the generalization of the Navier-Stokes equations. It is usually applied where the geometry is too complex to resolve with a mesh. The model can be used to simulate flows in rod or tube bundles, or between fins, where such effects are important, because both advection and diffusion terms are retained by the model. During the derivation of the continuum equations, the infinitesimal control volumes and surfaces are assumed relatively large to the inter- stitial spacing of the porous medium, but on the other hand they are small to the applied scales that are wanted to be resolved. Because of this consideration the control cells and control surfaces are assumed to contain both solid and fluid regions.

A generalization of Darcy's law for different Reynolds numbers is the Forchheimer equation. In ANSYS CFX for Directional Loss Model, it is implemented as follows:

$$
\begin{aligned}
& \frac{d P}{d x}=-\frac{\mu}{K_{\text {perm }(x)}} u_{x}-K_{\operatorname{loss}(x)} \frac{\rho}{2}|u| u_{x} \\
& \frac{d P}{d y}=-\frac{\mu}{K_{\text {perm }(y)}} u_{y}-K_{\operatorname{loss}(y)} \frac{\rho}{2}|u| u_{y} \\
& \frac{d P}{d z}=-\frac{\mu}{K_{\text {perm }(z)}} u_{z}-K_{\operatorname{loss}(z)} \frac{\rho}{2}|u| u_{z}
\end{aligned}
$$


where: $K_{\text {perm }}$ - permeability in different stream direction, $\mathrm{m}^{2} ; K_{\text {loss }}$ - quadratic loss coefficients in different stream direction, $1 / \mathrm{m} ; u$ - flow velocity in different direction, $\mathrm{m} / \mathrm{s} ; \mu$ - gas dynamic viscosity, $\mathrm{Pa} \cdot \mathrm{s} ; \rho$ - gas density, $\mathrm{kg} / \mathrm{m} 3$.

The linear component of Eqs. (1) - (3) represents viscous losses and the quadratic term represents inertial losses. Permeability and quadratic loss coefficients for different stream directions are defined as:

$$
\begin{gathered}
K_{\text {perm }(i)}=\frac{G \mu L_{i}}{A \rho \Delta P_{i}} \\
K_{l o s s(i)}=\frac{\Delta P_{i}}{\left(\frac{\rho u_{i}^{2}}{2}\right) L_{i}}
\end{gathered}
$$

where: $\mathrm{G}$ - gas flow rate, $\mathrm{kg} / \mathrm{m} 3$; $\mathrm{Li}$ - the length over which the pressure drop is taking place, $\mathrm{m}$; $\mathrm{A}$ - the crosssectional area to flow, $\mathrm{m} 2 ; \Delta \mathrm{Pi}$ - pressure drop, $\mathrm{Pa}$.

There is a critical value of the Reynolds number depending on the geometric characteristics of the porous medium. At low speeds, the inertial losses can be neglected and the equation will comply with Darcy's law. At high speeds, inertial losses will prevail. In this study, it is assumed that the viscous losses is negligible. But this issue requires further investigation.

Also, additional research requires a behavior at the interface of pure fluid domain and porous domain. ANSYS CFX only uses Darcy's equation in the porous domain. As a result, it does not ensure the continuity of shear stress at the interface of fluid domain and porous domain. In this case, calculation errors are possible.

\section{Method description}

The method proposed in this paper consists of the following main steps:

1. Full-scale fin modeling with the periodic boundary condition for several flow velocities (flow rates).

2. Determination of the heat transfer coefficient (HTC) and the loss coefficient of the fin for several flow velocities (flow rates).

3. Geometrical characteristics calculation of the finned tube (volume porosity, interfacial area density).

4. Representation of the finned region by a porous domain with the properties obtained in steps 2 and 3.

5. Modeling of a finned tube or tube bundle with a porous domain.

6. Verification and validation of porous medium model.

These steps are illustrated in the Fig. 1. At the first step, a fin unit cell domain with a periodic boundary condition is defined. We assume that hot gas flows external and cold gas flows inside of finned tube. Temperature and velocity for outside flow is specified at the inlet in the computational domain. The internal flow is defined by boundary conditions as a thermal convection. For that the temperature and the heat transfer coefficient of the finned tube inner wall are defined.

For the numerical simulations a three dimensional model of the finned tube are applied. A fine computational grid near the fin is required so that the parameter $\mathrm{Y}+$ is not higher than 5 . The grid must be checked for aspect ratios and skewing. Renormalization group theory (RNG) based $\mathrm{k}-\varepsilon$ turbulence model was used to calculate the turbulent flow.

In the second step, the average heat transfer coefficient is calculated as:

$$
H T C=\frac{Q}{T_{w}-T_{r e f}}
$$

where: $\mathrm{Q}$ - total rate of heat transfer, J; Tw - average fin temperature, K; Tref - reference temperature, $\mathrm{K}$. These values are determined from CFD analysis. 
The reference temperature in Eq. (6) is the average temperature of the hot gas between the inlet and outlet of the fin unit cell domain. The loss coefficient according to the Weisbach formula is calculated by Eq. (5). Pressure drop $\Delta \mathrm{Pi}$ is determined from CFD analysis for the unit cell domain.

In the third step, porosity and interfacial area density are calculated using simple formulas based on the finned tube geometry or, for example, according to the formulas given in [6].

In the fourth and fifth step to define porous medium properties (average HTC, loss coefficient, volume porosity and interfacial area density) in the finned tube. The principle of representation of the finned region by a porous domain shown in the Fig. 2.

In the sixth step, the finned tube model with the porous region is verified by comparing the outlet temperatures for fin unit cell domain (the hot gas flow section and flow rate proportionally change depending on the number of fins per unit length of the tube). Finally CFD analysis results is validated with experiment.

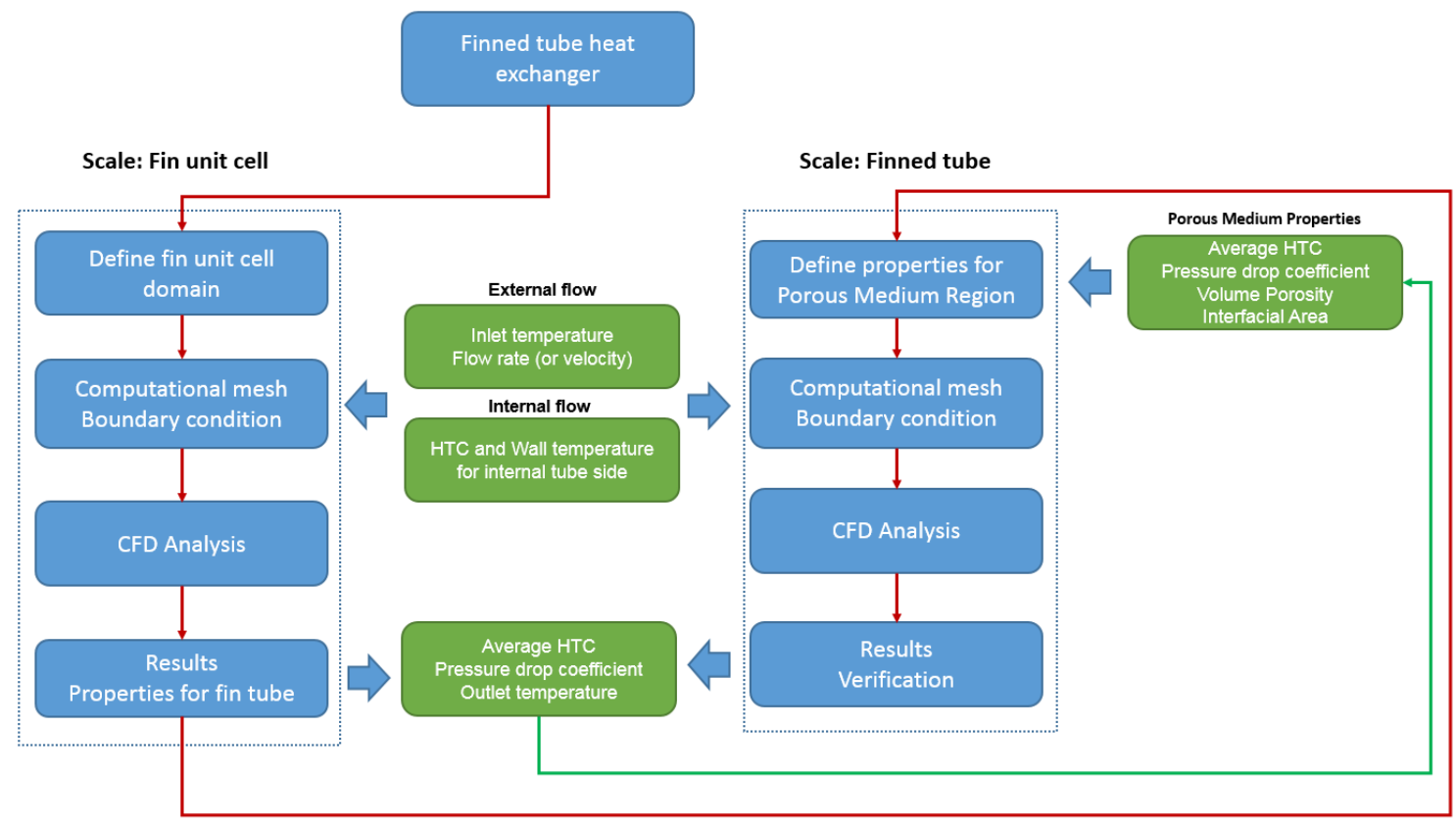

Fig. 1: Process diagram.

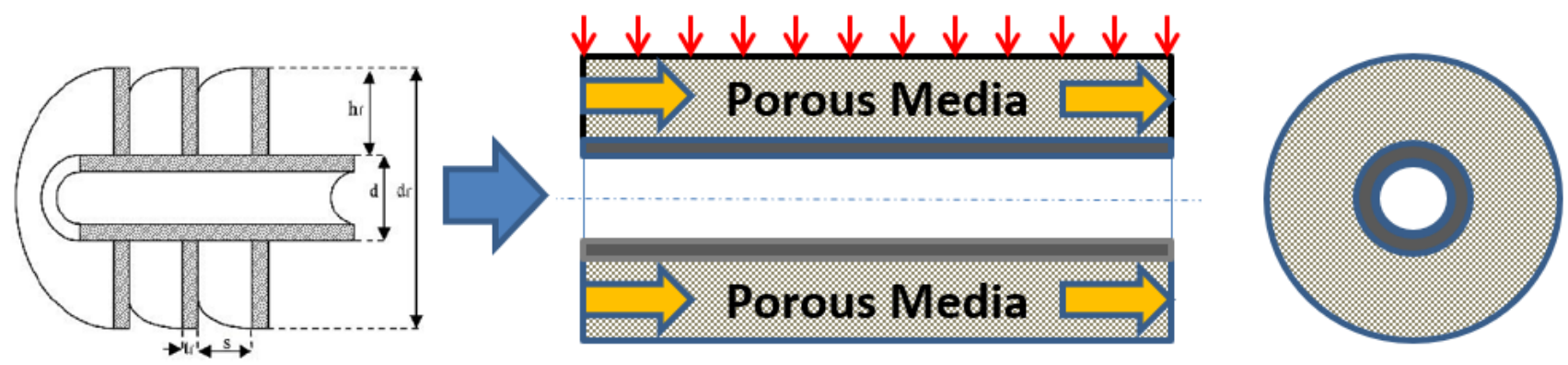

Fig. 2: The finned region Representation by a porous domain principle.

The heat transfer rate, outlet temperatures for gases and total pressure drop across the finned tube, as predicted by the CFD model, was compared with that obtained using several empirical correlations. Weierman, ESCOA and and Nir [14][16] were applied to all geometries (solid and serrated) and predict the total pressure drop, while Nir [16] predicts the 
friction factor. Robinson and Briggs [17] was used to predict pressure drop in the solid-fin, tube bundles. The Hofmann [18] and Næss [19] correlations were used to predict the friction factor for the serrated-fin tubes only.

The heat transfer rate and the total pressure drop could be calculated using the common equations and empirical correlations for described by Kays and London [20].

\section{Conclusion}

Method proposed in this paper permits to design heat-exchanger using porous medium approach. Suggested approach can be used in the science and industry. In further development method will be complete with dependences with thickness and numbers of fins and compare with experimental results. The heat transfer for aligned and staggered finned tube rows with a porous region will also be investigated.

\section{References}

[1] R. Hofmann, F. Frasz, and K. Ponweiser, "Heat Transfer and Pressure Drop Performance Comparison of FinnedTube Bundles in Forced Convection," WSEAS Trans. heat mass Transf., vol. 2, no. 4, pp. 72-88, 2007.

[2] H. Walter and R. Hofmann, "How can the heat transfer correlations for finned-tubes influence the numerical simulation of the dynamic behavior of a heat recovery steam generator?," Appl. Therm. Eng., vol. 31, no. 4, pp. 405-417, Mar. 2011.

[3] E. Martínez, W. Vicente, G. Soto, A. Campo, and M. Salinas, "Methodology for determining the optimal fin dimensions in helically segmented finned tubes," Appl. Therm. Eng., vol. 31, no. 10, pp. 1744-1750, Jul. 2011.

[4] M. M. Prieto, I. Suárez, F. J. Fernández, H. Sánchez, and C. Viescas, "Application of a thermal model to a power plant reheater with irregular tube temperatures," Appl. Therm. Eng., vol. 27, no. 1, pp. 185-193, Jan. 2007.

[5] M. M. Prieto, I. Suárez, F. J. Fernández, H. Sánchez, and M. Mateos, "Theoretical development of a thermal model for the reheater of a power plant boiler," Appl. Therm. Eng., vol. 27, no. 2-3, pp. 619-626, Feb. 2007.

[6] K. Hooman and H. Gurgenci, "Porous Medium Modeling of Air-Cooled Condensers," Transp. Porous Media, vol. 84, no. 2, pp. 257-273, 2010.

[7] Q. Zhang, S. Qin, and R. Ma, "Simulation and experimental investigation of the wavy fin-and-tube intercooler," Case Stud. Therm. Eng., vol. 8, no. 2013, pp. 32-40, 2016.

[8] U. Imke, "Porous media simplified simulation of single- and two-phase flow heat transfer in micro-channel heat exchangers," Chem. Eng. J., vol. 101, no. 1-3, pp. 295-302, 2004.

[9] M. Drahansky et al., "Computational Modeling of Vehicle Radiators Using Porous Medium Approach," Heat Exch. - Des. Exp. Simul., vol. 13, pp. 243-262, 2016.

[10] D. Juan and Z. Hai-Tao, "Numerical simulation of a plate-fin heat exchanger with offset fins using porous media approach," Heat Mass Transf. und Stoffuebertragung, vol. 54, no. 3, pp. 745-755, 2018.

[11] W. Wang, J. Guo, S. Zhang, J. Yang, X. Ding, and X. Zhan, "Numerical study on hydrodynamic characteristics of plate-fin heat exchanger using porous media approach," Comput. Chem. Eng., vol. 61, pp. 30-37, 2014.

[12] T. Brunschwiler et al., "Validation of the porous-medium approach to model interlayer-cooled 3D-chip stacks," 2009 IEEE Int. Conf. 3D Syst. Integr. 3DIC 2009, pp. 1-10, 2009.

[13] ANSYS CFX-Solver Theory Guide. 2011.

[14] Weierman and C., "Correlations Ease the Selection of Finned Tubes," Oil Gas J., pp. 94-100, 1976.

[15] V. Ganapathy, Industrial Boilers and Heat Recovery Steam Generators Design, Applications, and Calculations. New York: Marcel Dekker, Inc, 2003.

[16] A. Nir, "Heat transfer and friction factor correlations for crossflow over staggered finned tube banks," Heat Transf. Eng., vol. 12, no. 1, pp. 43-58, Jan. 1991.

[17] A. Bejan and A. Kraus, Heat transfer handbook. New Jersey: Jonh Wiley \& Sons, Inc., 2003.

[18] R. Hofmann and H. Walter, "Experimental and Numerical Investigation of the Gas Side Heat Transfer and Pressure Drop of Finned Tubes-Part II: Numerical Analysis," J. Therm. Sci. Eng. Appl., vol. 4, no. 4, pp. 1-11, 2012. 
[19] E. Næss, "Experimental investigation of heat transfer and pressure drop in serrated-fin tube bundles with staggered tube layouts," Appl. Therm. Eng., vol. 30, no. 13, pp. 1531-1537, Sep. 2010.

[20] W. M. Kays and A. L. London, Compact heat exchangers. New York: McGraw-Hill, 1984. 